\title{
A Deformable Convolutional Neural Network with Oriented Response for Fine-Grained Visual Classification
}

\author{
SHANGXIAN RUAN \\ Amazingx Academy, Foshan, China
}

\author{
JIATING YANG \\ Xian Institute of Optics and Precision \\ Mechanics, Chinese Academy of \\ Sciences, Xian, China
}

\author{
JIANBO CHEN* \\ University of Chinese Academy of \\ Sciences, Beijing, China
}

\begin{abstract}
Fine-grained visual classification (FGVC) aims to classify images belonging to the same basic category in a more detailed sub-category. It is a challenging research topic in the field of computer vision and pattern recognition in recent years. The existing FGVC method conduct the task by considering the part detection of the object in the image and its variants, which rarely pays attention to the difference in expression of many changes such as object size, posture, and perspective. As a result, these methods generally face two major difficulties: 1) How to effectively pay attention to the latent semantic region, and reduce the interference caused by many changes in pose and perspective; 2) How to extract rich feature information for non-rigid and weak structure objects. In order to solve these two problems, this paper proposes a deformable convolutional neural network with oriented response for FGVC. The proposed method can be divided into three main steps: firstly, the local region of latent semantic information is localized based on a lightweight CAM network; then, the deformable convolutional ResNet-50 network and the rotation-invariant coding oriented response network are designed, which input the original image and local region into the feature network to learn the discriminant features of rotation invariance; finally, the learned features are embed into a joint loss to optimize the entire network end-to-end. Experiments are carried out on three challenging FGVC datasets, including CUB-200-2011, FGVC_Aircraft and Aircraft_2 datasets. The results show that the accuracy of the proposed method on all datasets is better than the comparison method, which can effectively improve the accuracy of weakly supervised FGVC.
\end{abstract}

\section{CCS CONCEPTS}

- Computing methodologies $\rightarrow$ Artificial intelligence; Machine learning; • Theory of computation $\rightarrow$ Models of computation.

\section{KEYWORDS}

Fine-grained visual classification, Deformable convolution, Oriented response, Weakly supervised

\footnotetext{
* Jiating Yang is the corresponding author.

Permission to make digital or hard copies of all or part of this work for personal or classroom use is granted without fee provided that copies are not made or distributed for profit or commercial advantage and that copies bear this notice and the full citation on the first page. Copyrights for components of this work owned by others than ACM must be honored. Abstracting with credit is permitted. To copy otherwise, or republish, to post on servers or to redistribute to lists, requires prior specific permission and/or a fee. Request permissions from permissions@acm.org.

ICMLC '21, February 26-March 01, 2021, Shenzhen, China

(C) 2021 Association for Computing Machinery.

ACM ISBN 978-1-4503-8931-0/21/02 . \$ $\$ 15.00$

https://doi.org/10.1145/3457682.3457702
}

ACM Reference Format:

SHANGXIAN RUAN, JIATING YANG, and JIANBO CHEN. 2021. A Deformable Convolutional Neural Network with Oriented Response for FineGrained Visual Classification. In 2021 13th International Conference on Machine Learning and Computing (ICMLC '21), February 26-March 01, 2021, Shenzhen, China. ACM, New York, NY, USA, 8 pages. https://doi.org/10. $1145 / 3457682.3457702$

\section{INTRODUCTION}

Fine-grained visual classification (FGVC) is a very popular research topic in computer vision, pattern recognition and other fields in recent years [1]. It aims to classify images belonging to the same basic category (such as birds, aircrafts, dogs, etc.) into more detailed subcategories [2]. However, due to the greater intra-class difference, there are many variations in posture, viewing angle, occlusion, and background interference. In addition, the differences between classes are more detailed, so FGVC is a very challenging research task.

In the past ten years, most of FGVC methods are based on the detection of local distinguishable parts, looking for robust feature representations of images, which can be divided into four research contents: 1) fine-tuning methods based on conventional image classification networks, 2) method based on location detection and alignment, 3) method based on fine-grained feature learning, and 4) method based on visual attention mechanism. The first two types of methods above usually require additional object labeling information, such as a bounding box (Bbox), and the cost of obtaining manual labeling information is extremely high. Therefore, more and more scholars are increasingly focusing on the research of the latter two methods. Xiao et al. [3] propose a two-level attention algorithm, which is the first attempt to achieve FGVC by relying only on category tags. Since then, the research on weakly supervised FGVC has developed rapidly. The methods based on fine-grained feature learning mainly include: the bilinear convolutional neural network model (Bilinear-CNN) proposed by Lin et al. [4], which uses VGG-D (Visual Geometry Group-D) and VGG-M (Visual Geometry Group-M). The two networks are used as the basic network, and their output features are combined for classification to produce a better representation of fine-grained images; Zhang et al. [5] extract neural activations from convolutional neural networks, which are used to locate local regions and describe features, respectively. In addition, they propose a spatially weighted Fisher vector encoding method, which uses the spatially weighted combination of Fisher vectors to describe discriminative features. Wang et al. [6] propose an asymmetric multi-branch network, focusing on local and global information at the same time, and using saliency detection to extract the features of the saliency region. From the aspect 
of data amplification [7], Yang et al. propose a new self-supervising mechanism to learn discriminative subtle features.

Recently, research on FGVC based on visual attention mechanism has made a lot of progress. Aiming at the traditional attention model that only focuses on a single location, Zhao et al. [8] propose multiple attention networks, using Long Short-Term Memory (LSTM) networks to learn the region of attention and generate different attention regions at different times. Within each time step, it will predict the object category, and use the average of each prediction result to get the final prediction result. With the deepening of attention research, Sun et al. [9] propose a multi-attention and multi-constraint network, which combines metric learning and strengthen the connection between attention characteristics. Although the above methods have achieved certain results, they basically consider the part detection, and seldom pay attention to the differences in the expression of many changes, such as object size, posture, and perspective. The FGVC still has some difficulties, which are mainly reflected in: how to effectively pay attention to the latent semantic region, and reduce the interference caused by many changes in pose and perspective; how to extract rich objects for non-rigid and weak structure characteristic information.

In order to solve the above problems, inspired by the work done by Zhou et al. [10] on image region positioning and Zhou et al. [11], [12] on filter rotation learning, a deformable convolutional neural network with oriented response for FGVC. Different from the above methods, this paper first uses a lightweight localization network to locate the local region of the image with latent semantic information as a new input. Then, a deformable convolutional ResNet-50 network and a rotation-invariant coding direction response network (Oriented Response Network, ORN) are designed for feature extraction. Finally, features with less confusion, object deformation adaptation and rotation invariance are combined learning by fine-tuning the whole network.

The main works of this paper are as follows:

1) Based on the class activation map (CAM) [10] network, the local region with latent semantic information are localized.

2) Deformable convolutional ResNet-50 network structure and ORN network are designed to extract the local features and rotation invariant features of the object deformation adaptation.

3) The above features are embedded into an optimization objective of joint loss calculation, so that the integrated features are more discriminant.

The remaining sections of this paper are arranged as follows: Section 2 introduces some works related to this article. Section 3 presents the method proposed in this paper in detail. Section 4 introduces the experimental setup, presents and analyzes the experimental results. Section 5 gives the conclusion.

\section{RELATED WORKS}

The core task of FGVC research is to extract local discriminative subtle features, and generate feature representations with small intra-class differences and large inter-class differences. In recent years, with the rapid development of deep learning, FGVC methods have emerged from fine-grained feature learning to weakly supervised location detection methods. The following is a brief overview of fine-grained feature learning methods and weakly-supervised location detection sub-methods.

\subsection{Fine-Grained feature learning method}

The fine-grained features of objects are helpful for the classification and recognition of objects. Starting from finding local discriminative features, Liu et al. [14] propose a Fully Convolutional Attention Network (FCAN), which uses reinforcement learning based the visual attention model to locate multiple local regions of the object, and extracts the fine-grained features of the local region for classification. However, because the fixed-size attention region is used, background interference cannot be effectively avoided. The FGVC method based on local region positioning may lose discriminative small regions, such as bird claws and tail wings. The image classification method based on feature representation may extract features that are not sufficiently discriminative. Based on this, Fu et al. [15] propose Recurrent Attention Convolutional Neural Network (RA$\mathrm{CNN}$ ) to learn multi-scale Local region features, combining features of multiple scales for classification. The disadvantage is that the focus reflects in a multi-scale local region, and there may be greater redundancy between features. Based on an image focusing on a local region may not be enough to make a judgment, and focusing on multiple local regions at the same time can obtain more image information, Zheng et al. [16] propose a Multi-Attention Convolutional Neural Network (Multi-Attention Convolutional Neural Network, MA-CNN), using the different channels of the feature map to focus on different visual parts, clustering channels with similar peak responses to obtain the attention region. However, the feature alignment problem for multiple regions is worth exploring.

\subsection{Weakly supervised location detection method}

Among the methods based on component detection, Sermanet et al. [17] propose a deep recurrent neural network based on GoogLeNet. The network processes an image part at each time step to learn image features. Due to the redundancy between the image blocks and the useless background information, the final classification result is not ideal. Han et al. [18] propose a Squeeze-and-Excitation (SE) convolutional neural network based on the compression-stimulation module to strengthen the information channel and suppress useless channels to learn channel feature responses. It uses convolution filters to locate discriminative components, and learn the combination of part features and global features sort.

Different from all the above methods, this paper uses a lightweight CAM network to locate the local region with latent semantic information, and inputs it to the deformable convolutional ResNet50 network and the rotation-invariant coding ORN. Then, the local features and rotations adapted to the deformation unchanging characteristics are effectively extract. By combining intra-branch and inter-branch loss calculations to optimize the entire network, better distinguishing features can be extracted, and the classification performance of non-rigid, weakly structured objects, poses, and perspectives can be improved.

\section{PROPOSED METHOD}

Aiming at the problem that traditional neural network models based on attention mechanism cannot jointly focus on local features and rotation invariant features, this paper proposes a deformable convolutional neural network with oriented response for FGVC. First, the 


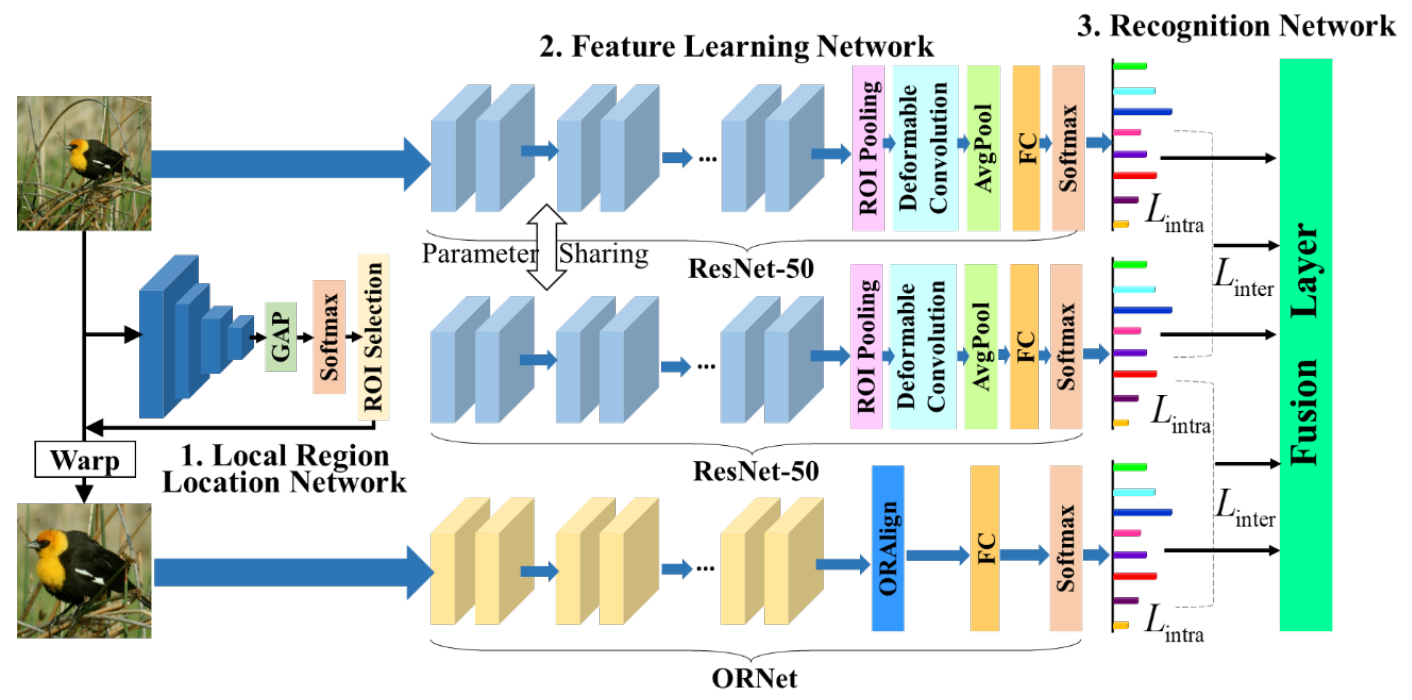

Figure 1: The Framework of the proposed method.

local region with latent semantic information is researched based on a lightweight CAM network; then, the deformable convolutional ResNet-50 network and the rotation-invariant encoding ORN are designed, and the original image and the local region are input to the feature network Perform feature extraction; finally, embed the learned features into a joint loss to optimize the entire network end-to-end for classification.

The overall framework of the method in this paper is shown in Figure 1, including two sub-networks: a local region positioning network and a feature extraction network. Among them, the last convolutional layer of the CAM positioning network and the global average pooling layer (Global Average Pooling, GAP) are used. Together with the Softmax classification layer, a class activation map is generated to locate the local region with latent semantic information (as shown in Figure 1). Then input the original image and the localized local region into two deformable convolutional ResNet-50 networks and rotation-invariant coding ORNs to form a three-branch feature network, and combine the loss and interbranch of the three branches Loss to a joint loss optimizes the entire network until convergence.

\subsection{CAM-Based local region location network}

As shown in Figure 1, in the CAM positioning network [10], the last convolutional layer is followed by the GAP layer and the Softmax classification layer. Map the predicted probability output by the Softmax layer back to the last convolutional layer to generate the class activation map. Specifically, for a given image, a global average value is calculated for each feature map at the GAP layer, and the average value of each feature map is output. Let $\mathrm{c}$ denote a certain category label, $f_{k}(x, y)$ denote the value of position $(x, y)$ on the $\mathrm{k}$-th feature map, and $w_{k}^{c}$ denote the weight of the c-th category on the $\mathrm{k}$-th feature map. After the two are multiplied, the activation feature map of the c-th category is obtained. Finally, the multiple activation feature maps are weighted to obtain the class activation map of the image. Define the class activation diagram belonging to a certain category c as:

$$
M_{c}(x, y)=\sum_{k} w_{k}^{c} f_{k}(x, y)
$$

Among them, $M_{c}(x, y)$ represents the weighting result of different feature maps to the recognition category $\mathrm{c}$, that is, the highlight region with the strongest response feature of the current image is located. Then select the local region with latent semantic information based on the gray threshold box, as shown in Figure 1, the Region of Interest Selection (ROI Selection) box is an added processing module, and finally the original image and ROI position information can be combined Get the cropped local region. The local region still retains a certain number of context pixels to help generate auxiliary information.

The CAM-based positioning network uses the GAP layer to replace the fully connected layer, which reduces network parameters, avoids over-fitting, and can locate local regions with potential semantic information. At the same time, the CAM network is lightweight, so there is no additional calculation overhead, and it has good adaptability.

\subsection{Feature learning network}

3.2.1 Deformable convolutional resnet-50 network. The traditional convolution kernel is affected by the fixed shape and can only collect local information in the fixed neighborhood, so it lacks the ability of adaptive feature expression. Deformable Convolution (Deformable Convolution) [18-19] uses expanded convolution to sample richer feature information in a larger neighborhood, but the output feature map still has the same spatial resolution.

This branch is based on the ResNet-50 network, in which each residual module is connected in series by three convolutional layers with convolution kernel sizes of $1 \times 1,3 \times 3$, and $1 \times 1$. Refer to the literature [19], as shown in Figure 1, we replace the 
$3 \times 3$ traditional convolution in the last convolution block with deformable convolution to form the ResNet- 50 of deformable convolution. Therefore, the original regular grid $\mathrm{R}$ will add an offset $\left\{\Delta p_{s}|s=1,2, \cdots, A|\right\}, A=|R|$. For each position $p_{0}$ in the output feature map $U, U\left(p_{0}\right)$ is calculated as:

$$
U\left(p_{0}\right)=\sum_{p_{s} \in R} w\left(p_{s}\right) \bullet I\left(p_{0}+p_{s}+\Delta p_{s}\right)
$$

where $I(\cdot)$ represents the input feature map. According to the principle of deformable convolution, firstly generate an offset at each position of the input feature map and propagate it forward; in the backward propagation stage, the newly added offset position $p_{s}+\Delta p_{s}$ will be learned at the same time, and then go through double Linear interpolation obtains the spatial position of the integer, and then performs deformable convolution.

It can be seen from the above that the deformable convolutional ResNet-50 network has increased the ability to detect object deformation and can extract richer discriminative features.

3.2.2 Rotation invariant coding direction response network. The difference within the fine-grained image category is even greater, and there are many changes in attitude, viewing angle, occlusion and background interference. In order to reduce the impact of changes within the class, this branch introduces ORN [11] for rotation invariant feature learning. As shown in Figure 1, all traditional convolutional layers are replaced with Oriented Response Convolution (ORConv), and a rotation-invariant alignment layer is connected after the last ORConv layer to form a rotation-invariant direction response network. The network first designs multiple Active Rotating Filters (ARF) in different directions to generate feature information in different directions. An ARF with O directions actively rotates $\mathrm{O}-1$ times during the convolution process to generate a feature map of $\mathrm{O}$ channels, which explicitly includes position and direction information. Based on the feature map $M$ of the active rotation filter $\mathrm{F}$ and $\mathrm{O}$ channels, the directional response convolution (ORConv) is defined as $\tilde{M}^{(k)}=\operatorname{ORConv}(F, M)$. The output feature map $\tilde{M}$ has O channels, and the $\mathrm{k}$-th channel is calculated as:

$$
\begin{gathered}
\tilde{M}^{(k)}=\sum_{o=0}^{O} F_{\theta_{k}}^{(o)} * M^{(o)} ; \\
\theta_{k}=k \frac{2 \pi}{O}, k=0,1, \cdots, O-1
\end{gathered}
$$

where $F_{\theta_{k}}^{(o)}$ is the active rotation filter $\mathrm{F}$ whose rotation angle in the o-th direction is $\theta_{k}$. The active rotation filter $\mathrm{F}$ in the 0 th direction can be regarded as physical, and it is learned through backward propagation, that is, the active rotation filter in the remaining O-1 directions (virtual) is used for updating, which is defined as:

$$
\begin{gathered}
F=F-\eta \sum_{k=0}^{O-1} \delta_{-\theta_{k}}^{(k)} ; \\
\delta^{(k)}=\frac{\partial L}{\partial F_{\theta_{k}}}, \theta_{k}=k \frac{2 \pi}{O}, k=0,1, \cdots, O-1
\end{gathered}
$$

where $\delta_{-\theta_{k}}^{(k)}$ represents the training error aligned to $-\theta_{k}$ in the kth direction, $\mathrm{L}$ represents the training loss, and $\eta$ represents the learning rate. According to formula (4), the main filter in the 0th direction is updated in the backward propagation stage, so the training errors of samples (blocks) with similar appearance but different directions are aggregated.
In ORN, the output features obtained through the above steps encode direction information, but they are not yet rotation-invariant. In order to obtain the invariant representation of the rotation within the class, this branch introduces the Oriented Response Align (ORAlign) layer to encode the above features. Specifically, an alignment method similar to Scale Invariant Feature Transform (SIFT) is used to find the direction with the strongest feature response and use it as the main direction, and then rotate the input feature to obtain the intra-class rotation invariant feature, and then Keep discriminative between classes.

Compared with Convolutional Neural Networks (CNN), ORN uses ARF to replace conventional filters, with fewer network parameters, can capture pattern features in more directions, and based on ORAlign layer processing to make the output features rotation invariant.

\subsection{Recognition network}

The method in this paper defines two loss functions: intra-branch loss and inter-branch loss. The intra-branch loss and the loss between adjacent branches are combined to optimize the network. The total loss function is defined as:

$$
L=\sum_{b=1}^{B} L_{\text {intra }}^{(b)}+\alpha \sum_{b=1}^{B-1} L_{\text {inter }}^{(b)}
$$

where $\mathrm{B}$ represents the number of branches, $\alpha$ is a factor that adjusts the relationship between the total loss and the loss of adjacent branches, as shown in the fusion layer in Figure 1. The intra-branch loss $L_{\text {intra }}^{(b)}$ uses the cross-entropy loss and is calculated as:

$$
L_{\text {intra }}^{(b)}=-\frac{1}{N} \sum_{k=1}^{N} \sum_{i=1}^{C} y_{i, k} \ln p_{i, k}
$$

where $\mathrm{N}$ represents the number of training samples, $\mathrm{C}$ is the number of categories, $y_{i, k}$ is the true category, and the probability that the $\mathrm{k}$-th sample is predicted to be the $\mathrm{i}$-th category is $p_{i, k}$. In order to ensure that the branches learn in a mutually reinforcing manner, the loss between branches is defined as:

$$
\begin{aligned}
L_{\text {inter }}^{(b)}= & \max \left(0, \sum_{k=1}^{N} Y_{k}\left(\ln P_{k}^{(b)}-P_{k}^{(b+1)}\right)+m\right) \\
& =\max \left(0, L_{\text {intra }}^{(b+1)}+m-L_{\text {intra }}^{(b)}\right)
\end{aligned}
$$

where $Y$ is the $y_{i, k}$ of all categories, and $P_{k}^{(\cdot)}$ is the $p_{i, k}$ of the designated branch of all categories. Each branch gradually learns its own feature representation with reference to adjacent branches, so that the overall loss error tends to be minimized and the classification performance is improved.

The convolution weight on the b-th branch is calculated by stochastic gradient descent as:

$w_{t+1}=w_{t}-\frac{\eta}{q} \sum_{j=1}^{q} \frac{\partial L_{j}}{\partial w_{t}}=w_{t}-\frac{\eta}{q} \sum_{j=1}^{q}\left(\left(1+\alpha \tau_{j}^{(b-1)}-\alpha \tau_{j}^{(b)}\right) \frac{L_{\text {intra, } j}^{(b)}}{\partial w_{t}}\right)$

where $\eta$ represents the learning rate, $L_{j}$ represents the loss value function of the j-th training sample, and q represents the batch size. $\tau$ represents the degree of association between adjacent branches 
Table 1: Data partitioning of the dataset

\begin{tabular}{cccc}
\hline Dataset & Class Number & Training Number & Testing Number \\
\hline CUB-200-2011 & 200 & 5994 & 5794 \\
FGVC_Aircraft & 100 & 6667 & 3333 \\
Aircraft_2 & 20 & 1333 & 667 \\
\hline
\end{tabular}

and is defined as:

$$
\tau_{j}^{(b-1)}=\left\{\begin{array}{lc}
1, & L_{\text {intra }}^{(b-1)}+m<L_{\text {intra }}^{(b)} \\
0, & \text { Otherwise }
\end{array}\right.
$$

If the $L_{\text {intra }}^{(b)}$ branch loss is significantly higher than the $L_{\text {intra }}^{(b-1)}$ branch loss, the learning rate $\alpha$ will increase to reduce the distance between branch $b$ and branch $b-1$. The entire network is optimized by training the total loss function, and the calculation results of different branches are merged to obtain the final prediction category.

\section{EXPERIMENT}

This section introduces experimental data and settings, experimental results and analysis, and ablation experiments.

\subsection{Dataset and experimental settings}

This paper uses two widely used and challenging image datasets CUB-200-2011 in the field of FGVC [20] and Fine-Grained Visual Classification of Aircraft (FGVC_Aircraft) [21] Experiment with the fighter dataset Aircraft_2 collected by a laboratory, and the summary is as follows:

CUB-200-2011: This dataset is the most challenging dataset in the field of FGVC. There are 200 images of different birds and a total of 11788 images. Due to the different attitudes and positions of birds in this dataset, the differences between classes are small and the differences within classes are large.

FGVC_Aircraft: The dataset has 102 different types of aircraft images, including civil aviation passenger planes, helicopters and drones. Each category includes 100 different images, for a total of 10200 images. This dataset has very detailed classification of aircraft models and high similarity between classes.

Aircraft_2: On the basis of the FGVC_Aircraft dataset, we collected 20 types of fighter images, each of which contains 100 images, for a total of 2000 images. Compared with the FGVC_Aircraft dataset, due to the different coatings and environments of fighters, there is a big difference in the class.

For the images in the CUB-200-2011 dataset, the size of each image ranges from 200 pixels $\times 200$ pixels to 500 pixels $\times 500$ pixels, and was adjusted to 448 pixels $\times 448$ pixels in the experiment. Each image in the FGVC_Aircraft dataset is approximately 800 pixels $\times$ 600 pixels, which is fixed at 800 pixels $\times 600$ pixels in the experiment. The image size of the dataset Aircraft_2 collected by the laboratory is about 600 pixels $\times 400$ pixels, and is uniformly adjusted to 600 pixels $\times 400$ pixels. The example images of the three fine-grained image datasets are shown in Figure 2, and the training set and test set of each dataset are divided as shown in Table 1

In the experiment, the convolutional layer parameters of the deformable convolution branch for offset learning are initialized to 0 .
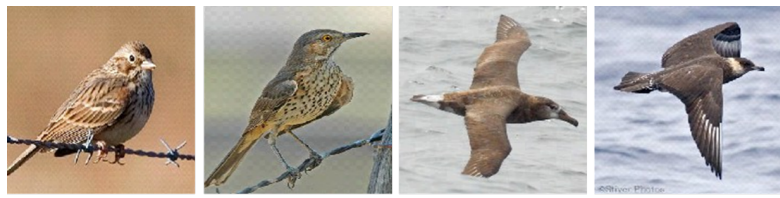

(a) CUB-200-2011
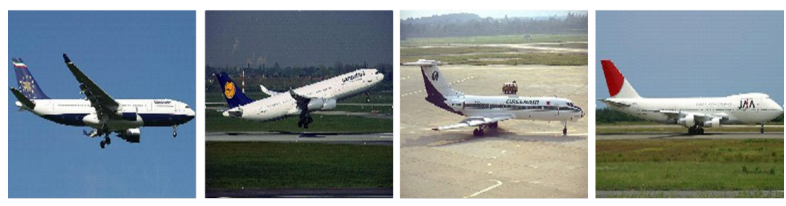

(b) FGVC Aircraft
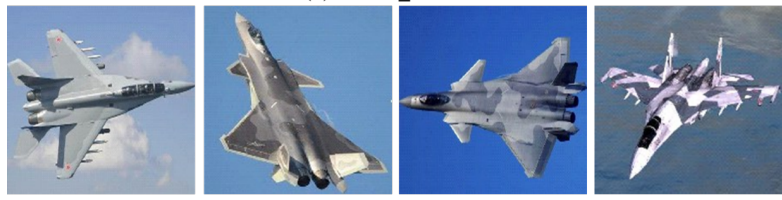

(c) Aircraft_2

Figure 2: Some examples of the adopted data samples.

$\alpha$ and $\mathrm{m}$ are set to 0.1 and 0.05 respectively. The batch size is uniformly set to 16 , the initial learning rate is 0.002 , and the iteration is 200 times. The learning rate is halved every 50 iterations. When the loss value remains stable, the optimal model is obtained. The layers of the deformable convolutional ResNe-50t network and the rotation-invariant encoding ORN are 50 and 18 respectively, and the pre-training models ResNet-50 and OR-ResNet-18 are used for initialization. The method in this paper is used for experimental simulation in Ubuntu 16.0 LTS system, using Pytorch-based deep learning framework, and using Tesla V100 graphics accelerator card for training.

\subsection{Comparison with the State-of-the-Arts}

In order to verify the classification performance of the method in this paper, a comparative experiment of different methods is first conducted on the CUB-200-2011 dataset. The experimental results are shown in Table 2. Part of the comparison method uses additional supervision information beyond the image category, including the bounding box (Bbox) provided by the dataset, and part labeling (Part). The other part adopts a weakly supervised attention mechanism. From the experimental results in Table 2, it can be seen that the classification accuracy of the proposed method has reached the same level compared with the previous strongly supervised classification methods. In addition, the classification accuracy of the proposed method is higher than that of multiple granularity CNN (MGCNN) 2.3 percentage points. At the same time, compared with 
Table 2: The result on the CUB-200-2011 dataset

\begin{tabular}{cccccccc}
\hline Method & Annotation & Attention & $\begin{array}{c}\text { Recognition } \\
\text { Accuracy(\%) }\end{array}$ & Method & Annotation & $\begin{array}{c}\text { Attention } \\
\text { Recognition } \\
\text { Accuracy(\%) }\end{array}$ \\
\hline Part-RCNN [22] & $\sqrt{ }$ & $\times$ & 76.4 & Bilinear-CNN[4] & $\times$ & $\times$ & 84.1 \\
MG-CNN [23] & $\sqrt{ }$ & $\times$ & 85.4 & FCAN [13] & $\times$ & $\sqrt{ }$ \\
Mask-CNN[12] & $\sqrt{ }$ & $\times$ & 87.3 & RA-CNN[14] & $\times$ & $\sqrt{ }$ & 84.3 \\
VGG-19 & $\times$ & $\times$ & 79.0 & MA-CNN[15] & $\times$ & $\sqrt{ }$ \\
ResNet-50 & $\times$ & $\times$ & 81.7 & Ours & $\times$ & $\sqrt{ }$ \\
\hline
\end{tabular}

Table 3: The result on the FGVC_Aircraft dataset

\begin{tabular}{cccc}
\hline Method & Annotation & Attention & Recognition Accuracy(\%) \\
\hline MG-CNN [23] & $\sqrt{ }$ & $\times$ & 86.6 \\
MDTP [24] & $\sqrt{ }$ & $\times$ & 85.4 \\
FV-CNN [25] & $\times$ & $\times$ & 81.5 \\
Bilinear-CNN [4] & $\times$ & $\times$ & 84.1 \\
RA-CNN [14] & $\times$ & $\sqrt{ }$ & 88.2 \\
MA-CNN [15] & $\times$ & $\sqrt{ }$ & 89.9 \\
Ours & $\times$ & $\mathbf{9 0 . 8}$ \\
\hline
\end{tabular}

the weakly supervised classification method that does not use additional labels, the proposed method has a significant improvement, which is 1.2 percentage points higher than the recent MA-CNN. This result proves that the proposed method can effectively extract complementary features and effectively distinguish fine-grained images (bird images with different sizes and few object pixels), since the proposed method adopts deformable convolutional neural network with oriented response, which can effectively pay attention to the latent semantic region.

According to the configuration described above, the weakly supervised fine-grained classification method based on the the proposed method has obtained a classification accuracy of $90.8 \%$ on the FGVC_Aircraft dataset. Table 3 shows the comparison results of different methods in this dataset. The classification accuracy of the proposed method is $0.9 \%$ higher than the recent MA-CNN, 5.4\% higher than the mining discriminative triplet of patch (MDTP) convolutional neural network. What's more, it is more superior than the fisher vector convolutional neural network (FV-CNN) about 9.3\%, proving the effectiveness of the proposed method. Similarly, some methods use additional information such as surrounding borders. The results on the FGVC_Aircraft dataset demonstrate the proposed method is more effective to learn discriminative features for FGVC than the existing state-of-the-art methods.

According to the configuration described above, the proposed method obtains $91.8 \%$ classification accuracy on the Aircraft_2 dataset collected in the laboratory. The other three comparison methods FCAN, RA-CNN and MA-CNN are all implemented in this experiment. It can be seen from the results in Table 4 that the proposed method has improved classification accuracy compared with other methods. At the same time, in terms of complexity, it can be seen that based on the use of the pre-training model, the proposed method can more effectively extract the features related to FGVC.
Since the airplane is a rigid object and has a clearer background, the classification accuracy is higher.

\subsection{Ablation Experiments}

In order to further verify the contribution of each branch of the proposed method, experiments are carried out on three different branches, including local region localization network (CAM), deformable convolutional network (DCN) and orientation response network (ORN). The experiment adopts the ResNet-50 network as the basic network (Baseline). The experimental results of different branches (or branch combinations) on the above three datasets are shown in Table 5

It can be seen from Table 5 that the proposed method is better than the classification results of a single branch (or two branches) methods and the baseline method on all datasets. Specifically, the classification accuracy of the proposed method is higher than the baseline method $6.0 \%, 4.1 \%$, and $4.1 \%$ on the three fine-grained datasets, respectively. This proves that the overall optimization of multi-branch neural networks can effectively extract important information that is helpful for classification. It can also be seen from the experimental results that the classification results of the deformable convolutional network (DCN) are slightly better than the local positioning network (CAM) and the directional response network (ORN), which is related to the number of layers of the network.

\section{CONCLUSION}

Considering that weakly-supervised FGVC is affected by many variations in semantic object pixel size, object pose and perspective, this paper proposes a deformable convolutional neural network with oriented response for weakly-supervised FGVC. The proposed method first uses the CAM network to quickly locate the local region with latent semantic information in the image, and 
Table 4: The result on the Aircraft_2 dataset

\begin{tabular}{cccr}
\hline Method & Annotation & Attention & Recognition Accuracy(\%) \\
\hline ResNet-50 & $\times$ & $\times$ & 87.7 \\
FCAN [13] & $\times$ & $\sqrt{ }$ & 87.5 \\
RA-CNN [14] & $\times$ & $\sqrt{ }$ & 89.1 \\
MA-CNN [15] & $\times$ & $\sqrt{ }$ & 90.3 \\
Ours & $\times$ & $\sqrt{ }$ & $\mathbf{9 1 . 8}$ \\
\hline
\end{tabular}

Table 5: The ablation results on the three datasets

\begin{tabular}{cccr}
\hline Method & CUB-200-2011 & FGVC_Aircraft & Aircraft_2 \\
\hline Baseline & 81.7 & 86.7 & 86.7 \\
CAM & 83.2 & 88.4 & 88.4 \\
DCN & 83.5 & 88.6 & 88.6 \\
CAM+DCN & 84.7 & 89.1 & 89.1 \\
ORN & 83.1 & 88.3 & 88.3 \\
Ours & $\mathbf{8 7 . 7}$ & $\mathbf{9 0 . 8}$ & $\mathbf{9 0 . 8}$ \\
\hline
\end{tabular}

inputs it to the deformable convolutional ResNet-50 network and the rotation-invariant coding ORN to learn the local features and rotation-invariant feature representations of the object deformation adaptation. Then, the learned features are embedded into a multi-branch loss calculation optimization objective, so as to obtain a more discriminative image representation. Evaluations on two publicly available fine-grained image datasets CUB-200-2011 and FGVC_Aircraft, and the fighter dataset Aircraft_2 collected by the laboratory verify the effectiveness of this method. The experimental results show that the proposed method is better than the comparison methods. However, in this study, we only consider the use of a single local region containing latent semantic objects in the image, and do not consider locating other regions of multiple objects in the image. In the next work, how to improve the model will be explored to make the method in this paper more perfect.

\section{ACKNOWLEDGMENTS}

The authors would like to thank all the people who kindly helped us in conducting this study. This work was supported by the Social Science Foundation and Student Work Research Project of Yangtze University under Grant 2018cxy13.

\section{REFERENCES}

[1] Chuanyi Zhang, Yazhou Yao, Xiangbo Shu, Zechao Li, Zhenmin Tang, and Qi Wu. 2020. Data-driven Meta-Set Based Fine-Grained Visual Recognition. In Proceedings of the 28th ACM International Conference on Multimedia (MM '20). Association for Computing Machinery, New York, NY, USA, 2372-2381. DOI:https://doi.org/10.1145/3394171.3414044.

[2] Yaxiong Chen, Xiaoqiang Lu, Shuai Wang. Deep Cross-Modal Image-Voice Retrieval in Remote Sensing. IEEE Transactions on Geoscience and Remote Sensing. 2020, pp. 1-13. doi: 10.1109/TGRS.2020.2979273.

[3] Tianjun Xiao, Yichong Xu, Kuiyuan Yang, Jiaxing Zhang, Yuxin Peng and Z. Zhang, The Application of Two-Level Attention Models in Deep Convolutional Neural Network for Fine-grained visual recognition, 2015 IEEE Conference on Computer Vision and Pattern Recognition (CVPR), Boston, MA, 2015, pp. 842-850, doi: 10.1109/CVPR.2015.7298685.

[4] Tsung-Yu Lin, Aruni RoyChowdhury, and Subhransu Maji, Bilinear CNN Models for Fine-Grained Visual Recognition, 2015 IEEE International Conference on Computer Vision (ICCV), Santiago, 2015, pp. 1449-1457, doi: 10.1109/ICCV.2015.170.
[5] Xiaopeng Zhang, Hongkai Xiong, Wengang Zhou, Weiyao Lin, and Qi Tian, Picking Neural Activations for Fine-Grained Recognition, IEEE Transactions on Multimedia, vol. 19, no. 12, pp. 2736-2750, Dec. 2017, doi: 10.1109/TMM.2017.2710803.

[6] Yaming Wang, Vlad I. Morariu, and Larry S. Davis, Learning a Discriminative Filter Bank Within a CNN for Fine-Grained Recognition, 2018 IEEE/CVF Conference on Computer Vision and Pattern Recognition, Salt Lake City, UT, 2018, pp. 4148-4157, doi: 10.1109/CVPR.2018.00436.

[7] Ze Yang, Tiange Luo, Dong Wang, Zhiqiang Hu, Jun Gao, and Liwei Wang, Learning to Navigate for Fine-Grained Classification, Proceedings of the European Conference on Computer Vision (ECCV), 2018, pp. 420-435, DOI:10.1007/978-3030-01264-9_26.

[8] Bo Zhao, Xiao Wu, Jiashi Feng, Qiang Peng, and Shuicheng Yan, Diversified Visual Attention Networks for Fine-Grained Object Classification, IEEE Transactions on Multimedia, vol. 19, no. 6, pp. 1245-1256, June 2017, doi: 10.1109/TMM.2017.2648498.

[9] Ming Sun, Yuchen Yuan, Feng Zhou, and Errui Ding, Multi-Attention Multi-Class Constraint for Fine-grained Image Recognition, Proceedings of the European Conference on Computer Vision (ECCV), 2018, pp. 805-821, DOI:10.1007/978-3030-01270-0 49.

[10] Bolei Zhou, Aditya Khosla, Agata Lapedriza, Aude Oliva, and Antonio Torralba, Learning Deep Features for Discriminative Localization, 2016 IEEE Conference on Computer Vision and Pattern Recognition (CVPR), Las Vegas, NV, 2016, pp. 2921-2929, doi: 10.1109/CVPR.2016.319.

[11] Yanzhao Zhou, Qixiang Ye, Qiang Qiu, and Jianbin Jiao, Oriented Response Networks, Proceedings of the IEEE Conference on Computer Vision and Pattern Recognition (CVPR), 2017, pp. 519-528, DOI:10.1109/CVPR.2017.527.

[12] Jue Wang, Wenchao Liu, Long Ma, He Chen, and Liang Chen, IORN: An Effective Remote Sensing Image Scene Classification Framework, IEEE Geoscience and Remote Sensing Letters, vol. 15, no. 11, pp. 1695-1699, Nov. 2018, doi: 10.1109/LGRS.2018.2859024.

[13] Xiao Liu, Tian Xia, Jiang Wang, Yi Yang, Feng Zhou, and Yuanqing Lin Fully Convolutional Attention Networks for Fine-Grained Recognition. arXiv preprint arXiv:1603.06765. 2016.

[14] Jianlong Fu, Heliang Zheng, and Tao Mei, Look Closer to See Better: Recurrent Attention Convolutional Neural Network for Fine-Grained Image Recognition, Proceedings of the IEEE Conference on Computer Vision and Pattern Recognition (CVPR), 2017, pp. 4438-4446, DOI: 10.1109/CVPR.2017.476.

[15] Heliang Zheng, Jianlong Fu, Tao Mei, and Jiebo Luo, Learning Multi-Attention Convolutional Neural Network for Fine-Grained Image Recognition, 2017 IEEE International Conference on Computer Vision (ICCV), Venice, 2017, pp. 5219-5227, doi: 10.1109/ICCV.2017.557.

[16] Pierre Sermanet, Andrea Frome, and Esteban Real, Attention for fine-grained categorization. arXiv preprint arXiv:1412.7054. 2014.

[17] Junwei Han, Xiwen Yao, Gong Cheng, Xiaoxu Feng, and Dong Xu, P-CNN: Part-Based Convolutional Neural Networks for Fine-Grained Visual Categorization, in IEEE Transactions on Pattern Analysis and Machine Intelligence, doi: 10.1109/TPAMI.2019.2933510.

[18] Jifeng Dai, Haozhi Qi, Yuwen Xiong, Yi Li, Guodong Zhang, Han Hu, and Yichen Wei, Deformable Convolutional Networks, Proceedings of the IEEE 
International Conference on Computer Vision (ICCV), 2017, pp. 764-773, DOI:10.1109/ICCV.2017.89.

[19] Xizhou Zhu, Han Hu, Stephen Lin, and Jifeng Dai, Deformable ConvNets V2 More Deformable, Better Results, Proceedings of the IEEE/CVF Conference on Computer Vision and Pattern Recognition (CVPR), 2019, pp. 9308-9316

[20] Wah, C., Branson, S., Welinder, P., Perona, P., \& Belongie, S. (2011). The caltechucsd birds-200-2011 dataset.

[21] Subhransu Maji, Esa Rahtu, Juho Kannala, Matthew Blaschko, Andrea Vedaldi, Fine-Grained Visual Classification of Aircraft, arXiv preprint arXiv:1306.5151. 2013.

[22] Ning Zhang, Jeff Donahue, Ross Girshick, and Trevor Darrell, Part-Based R-CNNs for Fine-Grained Category Detection. In: Fleet D., Pajdla T., Schiele B., Tuytelaars
T. (eds) Computer Vision - ECCV 2014. ECCV 2014. Lecture Notes in Computer Science, vol 8689. Springer, Cham. https://doi.org/10.1007/978-3-319-10590-1_54. [23] Yaxiong Chen, Xiaoqiang Lu, and Xuelong Li. Supervised deep hashing with a joint deep network. Pattern Recognition. 2020, 105. 107368. doi: 10.1016/j.patcog.2020.107368.

[24] Yaming Wang, Jonghyun Choi, Vlad Morariu, Larry S. Davis, Mining Discriminative Triplets of Patches for Fine-Grained Classification, Proceedings of the IEEE Conference on Computer Vision and Pattern Recognition (CVPR), 2016, pp. 1163-1172, DOI: 10.1109/CVPR.2016.131.

[25] Philippe-Henri Gosselin, Naila Murray, Hervé Jégou, and Perronnin, Florent, Revisiting the Fisher Vector for Fine-Grained Classification, Pattern Recognition Letters, 49, 92-98, https://doi.org/10.1016/j.patrec.2014.06.011. 